\title{
Births Averted by Contraception: An Approach Through Renewal Theory
}

\author{
R. G. PotTeR \\ Department of Sociology and Anthropology, Brown University, \\ Providence, Rhode Island 02912 \\ and Population Studies Center, University of Michigan. \\ Ann Arbor, Michigan 48104 \\ Received December, 1969
}

\section{INTRODUCTION}

Of critical interest to family planning administrators are estimates of births averted per segment of contraception. By a "segment" of contraception is meant the period of usage of a method between its initiation (or resumption after a period of nonuse) and its next interruption owing to accidental pregnancy, switch to another method, or stopping contraception. Several attempts to estimate births averted per IUD inserted have been published. ${ }^{1}$ These analyses have been too tailored to specific data and too empirical to provide an ideal vehicle for clarifying the conditions under which births averted per segment of contraception is high or low. Nevertheless, these applied analyses have served to establish some of the factors that deserve place in a theory of family planning impact.

Because they provide such efficient protection against pregnancy, modern contraceptives such as the IUD or the antiovular pills have an impact that is limited less by accidental pregnancy than by high discontinuation rates for other reasons. Another qualifying factor is the amount of overlap between practice of the contraceptive and the anovulatory period following childbirth when the woman is protected anyway. Even when all these factors are held

${ }^{1}$ The pioneering analysis is that of LeE, B. M. AND IsBISTER, J. 1966. The impact of birth control programs on fertility, in "Family Planning and Population," (B. Berelson et al., Eds.), pp. 737-758, Univ. Chicago Press, Chicago, Ill. Two analyses which are similar in the factors they include are: WoLFERs, D. 1969. The demographic effects of a contraception programme, Population Studies 23, 111-140; PotTER, R. G. 1969. Estimating births averted in a family planning program, in "Fertility and Family Planning: A World View," (S. J. Behrman, L. Corsa, and R. Freedman, Eds.), pp. 413-434, Univ. Michigan Prcss, Ann Arbor, Mich. A number of procedures are reviewed in MAULdiN, W. P. 1968. Births averted by family planning programs, Studies in Family Planning, No. 33, 1-6.

(C) 1970 by Academic Press, Inc. 
constant, the impact of a contraceptive depends closely on the potential fertility of its users, that is, the fertility that they might have exhibited had they not adopted the method in question. This potential fertility depends on whether the method is substituting or not for another contraceptive and the characteristics of the latter. In turn, the degree and nature of substitution is closely related to the family planning setting. Is the family planning program introducing new contraception into a community; is it persuading couples to undertake familiar procedures of family limitation earlier in their reproductive careers; or it is merely encouraging clients to shift from one program contraceptive to another? It will be shown that the impact of the identical contraceptive varies in the three contexts.

Presented below is an analytical model that yields estimates of births averted by one segment of contraception as a function of the factors just mentioned. The approach is to compare pairs of renewal processes representing two cohorts of couples alike in all respects except that during an initial interval one cohort exploits the contraceptive under assessment while the other does not. A qualitative statement of this approach has been given elsewhere (Potter, 1969). Its mathematical basis is the renewal process of Perrin and Sheps and for background the reader may wish to refer to their basic paper (Perrin and Sheps, 1964; Sheps, 1963; Sheps, Menken, and Radick, 1969). The formulation of Perrin and Sheps, which allows only for terminating contraception because of accidental pregnancy, has been extended to include discontinuation of the contraceptive for other reasons.

The present model does not include such factors as sterility, mortality, or age-related changes in fecundity. Therefore it is realistic only for the first half of the childbearing period when sterility is infrequent and changes in fecundity are slow. Applications of the model, considered in the last section of the paper, are chosen accordingly.

The approach of comparing pairs of renewal processes may be adapted for investigating the relative impact of induced abortion and contraception. That task is reserved for another paper.

\section{NOMENCLATURE}

For the reader's convenience a glossary of terms is provided below. When possible the nomenclature of Perrin and Sheps is followed, though with one modification. Extra subscripts are employed when it is desired to mark the absence of contraception or identify the contraceptives being used. Specifically, the appended subscript " $f$ " signifies natural fertility while one or an ordered pair of the subscripts " $c$," " $x$," and " $y$ " indicate the presence and sequencing of segments of the particular contraceptives $C, X$, and $Y$. 
f

$\theta_{i}$

$v_{i}$

$\xi_{i}^{2}$

$u_{i 0}$

$\sigma_{i, 1}^{2}$

$\eta$

$\lambda_{i}^{2}$

$u_{\mathrm{v1}}, \sigma_{01}^{2}$

$u_{11}, \sigma_{11}^{2}$

$u_{44}, \sigma_{44}^{2}$

$E\left[N_{i}(T) \mid S_{j}\right]$

$T_{i j}$

$T_{i j}^{*}$

$T_{11, \overline{4}}$

$e_{x}$

$p$

A

$d_{x}$

$d_{x}$

$k_{x}$

$k_{x y}$

$k_{x y f}$

$u_{01 . f}$

$u_{01, z}$

$u_{01, x y}$

I

B
Natural fecundability

Probability of pregnancy ending in outcome $i$

$i=2$ abortion or miscarriage;

$i=3$ stillbirth;

$i=4$ live birth.

Mean length of pregnancies of outcome $i$

Corresponding variance

Mean length of anovulatory periods following pregnancies of outcome $i$ Corresponding variance

$v_{i}+u_{i 0}$

$\xi_{i}^{2}+\sigma_{i 11}^{2}$

Mean and variance of delay to next conception

Mean and variance of the pregnancy interval

Mean and variance of the birth interval state $j$

Random time required for passage from state $S_{i}$ to $S_{j}$

Random length of stay in the ith state when the next state entered is $S_{j}$

Time spent in the recurrence of pregnancy accomplished without passage through state 4

Effectiveness of contraceptive $X$

$\left(1-e_{x}\right) f$, the monthly risk of conception during a fecundable month when practicing $X$

Interval from initiation of contraception to end of anovulatory period

Monthly probability of discontinuing $X$ during an anovulatory month

$\left(1-p_{x}\right) d_{x}$, monthly probability of discontinuing $X$ for a reason other than accidental pregnancy during a fecundable month

Proportion practicing $X$ at start of fecundable period

Proportion practicing $Y$, having already dropped $X$, at start of fecundable period

Proportion unprotected by contraception, having dropped first $X$ and then $Y$, at start of fecundable period

Expected delay to next conception in absence of contraception

Expected delay to next conception in presence of one segment of $X$

Expected delay to next conception, given one segment of $X$ followed conditionally by one segment of $Y$

Expected net delay to next conception, (always with two subscripts, the first indicating the experimental condition, the second subscript the control condition)

Births averted per segment of contraception 


\section{Basic Model}

As originally formulated by Perrin and Sheps, during any month a woman is in one of five possible states:

$S_{0}$-fecundable and subject to a constant monthly risk of conception;

$S_{1}$ - pregnant;

$S_{2}$-infecundable owing to the temporary anovulation that follows abortion or miscarriage;

$S_{3}$-anovulatory following a stillbirth;

$S_{4}$-anovulatory following a live birth.

Possible transitions are from the fecundable state $S_{0}$ to pregnancy $S_{1}$ and from pregnancy through one of the temporary anovulatory states $-S_{2}, S_{3}$, or $S_{4}$ - to the fecundable state again. The family-building history of a woman (i.e., timing of her pregnancies and births) is determined by the sequence of states visited and the length of time spent in each. "For example, the number of live births produced by a woman in time $T$ is given by the number of transitions from $S_{1}$ to $S_{4}$ in that time, the total number of pregnancies entered is given by the number of transitions from $S_{0}$ to $S_{1}$, etc." (Perrin and Sheps, 1964, p. 31).

Following Perrin and Sheps, we let $T_{i j}$ designate the random time in months required for passage from $S_{i}$ to $S_{j}$, this passage time having a mean of $u_{i j}$ and variance $\sigma_{i j}^{2} . T_{i j}^{*}$ denotes the random length of stay in the $i$-th state when the next state entered is $S_{j}$.

Natural fecundability, the monthly probability of conceiving during a fecundable month in the absence of contraception, is denoted by $f$. Length of stay in the pregnant state $T_{1 i}^{*}, i=2,3,4$, is governed by a probability distribution conditional to outcome of pregnancy $i$ with mean $v_{i}$ and variance $\xi_{i}{ }^{2}$. Length of stay in the postdelivery anovulatory period $T_{i 0}, i=2,3,4$, is governed by a probability distribution conditional to outcome of pregnancy $i$ with mean $u_{i 0}$ and variance $\sigma_{i 0}^{2}$. It is assumed that for a given pregnancy outcome, lengths of pregnancy and lengths of the following anovulatory periods are independently distributed. It is convenient to have symbols for the first two moments of the total infecundable period $T_{1 i}^{*}+T_{i 0}^{*}$ which are $\eta_{i}=v_{i}+u_{i 0}$ and $\lambda_{i}{ }^{2}=\xi_{i}{ }^{2}+\sigma_{i 0}^{2}$. The probabilities that a pregnancy will end in abortion, stillbirth, or live birth are designated $\theta_{2}, \theta_{3}$, and $\theta_{4}$ respectively. It is understood that $\theta_{2}+\theta_{3}+\theta_{4}=1.0$.

To qualify as a renewal process, the family-building process must be formulated in such a way that intervals between consecutive births, or between consecutive pregnancies, behave as independent, identically distributed random variables. In a so-called "augmented renewal process," the initial interval (e.g., from marriage to first birth) may be distributed differently than subsequent intervals but must be distributed independently of them. 
To meet these qualifications, it has proven necessary to accept three simplifying assumptions:

(a) Homogeneity among women (the same probabilities apply to all women);

(b) Homogeneity in time (all parameters are fixed in time and therefore are independent of age);

(c) The reproductive period is "sufficiently long" (i.e., infinite).

The power of the model is revealed in four kinds of results. First are moments of first passage and recurrence times. Examples are the means and variances of the waiting time to next conception, $u_{01}$ and $\sigma_{01}^{2}$; of the interval between consecutive pregnancies, $u_{11}$ and $\sigma_{11}^{2}$; and of the interval between consecutive live births, $u_{44}$ and $\sigma_{44}^{2}$.

Given homogeneity in time and among women, then with a sufficient passage of time, the proportion of women in each possible state stabilizes, resulting in a constant birth rate which can be proven to equal the reciprocal of the expected birth interval, or $\boldsymbol{u}_{\mathbf{4 4}}^{-1}$. Hence, a second type of result is the equilibrium proportions in each of the possible five states. A third result is the birth rate (or pregnancy rate) characteristic of the process upon its reaching equilibrium.

A fourth result is the linear counting functions $E\left[N_{i}(T) \mid S_{j}\right]$ that give the expected number of pregnancies $(i=1)$ or of births $(i=4)$ during a specified duration $T$ if the process starts during a fecundable month $(j=0)$, at the start of a pregnancy $(j=1)$, or right after a pregnancy of outcome $j=2,3$, or 4 . For example, the number of births to be expected in a duration of $T$ months if all women start in state $S_{j}$ is

$$
E\left[N_{4}(T) \mid S_{j}\right]=\frac{T}{u_{44}}+\frac{u_{44}^{(2)}+u_{44}}{2 u_{44}^{2}}-\frac{u_{j 4}}{u_{44}},
$$

where $u_{44}^{(2)}$ is the second moment around the origin of the birth interval $T_{44}$ and $u_{j 4}$ denotes the expected interval from entrance into state $S_{j}$ to next birth. Note that this expected number of births is a linear function in $T$. The corresponding formula for number of pregnancies is analogous, with "1" replacing " 4 " in the appropriate subscripts. These linear functions are approximate unless $T$ is sufficiently large to allow the process to reach equilibrium.

\section{Some Formulas Derived}

Explicit formulas are now derived for delays to next conception in the presence of a single segment of contraceptive $X$. What has been called a passage time $T_{01}$ from the fecundable state $S_{0}$ to the pregnancy state $S_{1}$ will henceforth 
be termed a conceptive delay. Being measured is the number of fecundable months preceding, but not including the month of conception.

In their treatment of contraception, Perrin and Sheps provide for a risk of accidental pregnancy during practice of contraception, but not for discontinuing it for other reasons. In the extension of their renewal process considered below, any contraceptive $X$ is associated with two parameters. First is its "effectiveness" $e_{x}, 0 \leqslant e_{x} \leqslant 1$, whereby during its practice the monthly risk of pregnancy during a fecundable month is reduced from the woman's natural fecundability $f$ to a "residual fecundability" $p_{x}=\left(1-e_{x}\right) f$. Second is the monthly risk $d_{x}$ ' of stopping use for a reason other than pregnancy.

The following additional simplifying assumption is allowed: contraception is initiated at the start of the month; ovulation and therefore conception occurs in the middle of the month; and discontinuation of contraception for a reason other than pregnancy always occurs at the end of the month. These conventions mean that users of $X$ during an anovulatory month are exposed to the single risk $d_{x}{ }^{\prime}$ of dropping $X$ at the end of the month. Among users during a fecundable month, $p_{x}$ will accidentally conceive, $d_{x}=\left(1-p_{x}\right) d_{x}{ }^{\prime}$ will drop $X$ at the end of the month, and $1-p_{x}-d_{x}$ will continue its use into the next month.

It is also necessary to specify a parameter $A$ representing the interval in months between initiation of $X$ and end of the anovulatory period. When $X$ is commenced several months before the end of this anovulatory period and a fraction of women drop $X$ to enter the fecundable period unprotected, the impact of $X$ is lessened.

Additional subscripts " $f$ " and " $x$ " will be used to distinguish between absence of contraception and presence of one segment of the contraceptive $X$. When useful, the value of parameter $A$ will be indicated in parentheses. For example, the mean and variance of conceptive delay in the absence of contraception is denoted $u_{01, f}$ and $\sigma_{01, f}^{2}$, whereas the same moments given a single segment of $X$ initiated $A$ months before end of anovulation are signified by $u_{01 . x}(A)$ and $\sigma_{01, x}^{2}(A)$.

Of course the simplest case is that of no contraception. Because the monthly chance of conception during a fecundable month is constant, conceptive delays behave as a geometrically distributed random variable with parameter $f$. Thus, it is readily shown that

and

$$
\begin{aligned}
u_{01, f} & =(1-f) f+2(1-f)^{2} f+\cdots \\
& =(1-f) / f
\end{aligned}
$$

$$
\sigma_{01, f}^{2}=(1-f) / f^{2} \text {. }
$$

The second moment of $T_{01, f}$ around the origin, denoted as $u_{01, f}^{2}$, is

$$
u_{01, f}^{(2)}=\sigma_{01, f}^{2}+\left(u_{01, f}\right)^{2} .
$$


Note that if one is concerned not with months of conceptive delay, but months during which conception is a possibility, one has

$$
f+2(1-f) f+3(1-f)^{2} f+\cdots=1 / f,
$$

and the same variance as before, namely, $(1-f) / f^{2}$.

We consider next $u_{01, x}(0)$, the conceptive delay expected when a single segment of $X$ is initiated at the start of the first fecundable month. The ratio of accidental pregnancies to discontinuations for other reasons, namely, $p_{x}$ to $d_{x}$, remains the same from one fecundable month to another. Hence the proportion eventually conceiving under $X$ is $p_{x} /\left(d_{x}+p_{x}\right)$ and the proportion ultimately dropping $X$ for other reasons is $d_{x} /\left(d_{x}+p_{x}\right)$. Moreover the following argument shows that these two groups have the same mean duration of $X$-use.

Among those women who conceive during practice of $X$, the proportions accidentally conceiving during months $1,2, \ldots, i$, are

$$
\begin{aligned}
& \frac{p_{x}}{p_{x} /\left(d_{x}+p_{x}\right)}, \frac{\left(1-d_{x}-p_{x}\right) p_{x}}{p_{x} /\left(d_{x}+p_{x}\right)}, \ldots, \frac{\left(1-d_{x}-p_{x}\right)^{i-1} p_{x}}{p_{x}\left(d_{x}+p_{x}\right)}, \ldots \\
& -\left(d_{x}+p_{x}\right),\left(1-d_{x}-p_{x}\right)\left(d_{x}+p_{x}\right), \ldots,\left(1-d_{x}-p_{x}\right)^{i-1}\left(d_{x}+p_{x}\right), \ldots
\end{aligned}
$$

which is the probability function of a geometrically distributed random variable with parameter $d_{x}+p_{x}$. The mean and variance of the number of months of $X$-practice is therefore $1 /\left(d_{x}+p_{x}\right)$ and $\left(1-d_{x}-p_{x}\right) /\left(d_{x}+p_{x}\right)^{2}$, respectively.

Among those women who conceive after discontinuing $X$, the proportions who drop $X$ after $1,2, \ldots, i, \ldots$ months of use are

$$
\frac{d_{x}}{d_{x} /\left(d_{x}+p_{x}\right)}, \frac{\left(1-d_{x}-p_{x}\right) d_{x}}{d_{x} /\left(d_{x}+p_{x}\right)}, \ldots, \frac{\left(1-d_{x}-p_{x}\right)^{i-1} d_{x}}{d_{x} /\left(d_{x}+p_{x}\right)}, \ldots
$$

which after cancellation yields the same probability function as before, thereby proving that the two groups share the same distribution of $X$-use.

It follows, then, that for women conceiving during practice of $X$, mean months of conceptive delay are

$$
\frac{1}{d_{x}+p_{x}}-1
$$

and for women conceiving after stopping $X$, the mean delay is

$$
\frac{1}{d_{x}+p_{x}}+\frac{1-f}{f}
$$

The homogeneity of the population with respect to $e_{x}$ and $f$ insures that lengths of $X$ practice and delays of conception after stopping $X$ are stochastically 
independent. The above two expected delays properly weighted yield as the mean conceptive delay for the total population

$$
u_{01, x}(0)=\left(\frac{p_{x}}{d_{x}+p_{x}}\right)\left(\frac{1}{d_{x}+p_{x}}-1\right)+\left(\frac{d_{x}}{d_{x}+p_{x}}\right)\left(\frac{1}{d_{x}+p_{x}}+\frac{1-f}{f}\right) .
$$

Now let $A>0$. The proportion of women who may be expected to retain $X$ into the fecundable period is

$$
k_{x}=\left(1-d_{x}{ }^{\prime}{ }^{A}\right.
$$

The complement, $1-k_{x}$, drop $X$ during anovulation. The expected conceptive delay of the latter is $(1-f) / f$ while for the former it is $u_{01, x}(0)$, derived above. Hence, the mean conceptive delay for the total group is

$$
u_{01, x}(A)=k_{x} u_{01, x}(0)+\left(1-k_{x}\right)(1-f) / f .
$$

To obtain the variance $\sigma_{01, x}^{2}(A)$, it is easiest to derive first the second moment around the origin $u_{01, x}^{(2)}(A)$ and then, having already the mean $u_{01, x}(A)$, calculate the variance by the relation

$$
\sigma_{01, x}^{2}(A)=u_{01, x}^{(2)}(A)-\left(u_{01, x}(A)\right)^{2} .
$$

We proceed by subdividing the population into three subpopulations, each corresponding to a distinct pairing of statuses at start of the fecundable period and at time of conception. The identifications are given in the first two columns

\begin{tabular}{|c|c|c|c|c|}
\hline \multicolumn{3}{|c|}{ Identification of Subpopulation } & \multicolumn{2}{|c|}{ Conceptive Delay } \\
\hline $\begin{array}{c}\text { Status at } \\
\text { Start of } \\
\text { Fecundable } \\
\text { Period }\end{array}$ & $\begin{array}{l}\text { Status at } \\
\text { Time of } \\
\text { Conception }\end{array}$ & Weight & Mean & Variance \\
\hline Using $X$ & Using $X$ & $k_{x}\left(\frac{p_{x}}{d_{x}+p_{x}}\right)$ & $\frac{1}{d_{x}+p_{x}}-1$ & $\frac{1-d_{x}-p_{x}}{\left(d_{x}+p_{x}\right)^{2}}$ \\
\hline Using $X$ & Unprotected & $k_{x}\left(\frac{d_{x}}{d_{x}+p_{x}}\right)$ & $\frac{1}{d_{x}+p_{x}}+\frac{1-f}{f}$ & $\frac{1-d_{x}-p_{x}}{\left(d_{x}+p_{x}\right)^{2}}+\frac{1-f}{f^{2}}$ \\
\hline Unprotected & Unprotected & $1-k_{x}$ & $\frac{1-f}{f}$ & $\frac{1-f}{f^{2}} \quad 1-f$ \\
\hline
\end{tabular}
of Table I. The next three columns give the weight of the subpopulation in the

TABLE I

Components in the Calculation of $u_{01, x}^{(2)}(A)$ 
total population, the mean delay to next conception of the subpopulation, and the corresponding variance. In each row the sum of the variance plus the mean squared yields the second moment around the origin (not shown in Table I). Finally the sum of products of these second moments around the origin times their weights gives the desired $u_{01, x}^{(2)}(A)$.

In the special case $A=0$, all women start the fecundable period practicing $X$. That is, $k_{x}=1.0$ and the bottom row of Table I vanishes.

We now consider the case where $X$ is adopted $A$ months before end of anovulation and if $X$ is dropped for a reason other than pregnancy, a second contraceptive $Y$ of characteristics $e_{y}$ and $d_{y}{ }^{\prime}$ is immediately adopted. If $Y$ is dropped, the woman remains unprotected until conception. The mean, variance, and second moment around the origin of conceptive delays we denote as

$$
u_{01, x y}(A), \sigma_{01, x y}^{2}(A), \text { and } u_{01, x y}^{(2)}(A) .
$$

This time we have to partition the population into six subpopulations, each corresponding to an unique pairing of statuses at the start of the fecundable period and at time of conception. See Table II.

At the start of the first fecundable month, a woman may be practicing $X$, practicing $Y$, or under a regime of natural fecundability.

We use the following notation:

$k_{x}$-proportion who start the fecundable period practicing $X$,

$k_{x y}$-proportion who start the fecundable period practicing $Y$, having dropped $X$,

$k_{x y f}$-proportion who start the fecundable period subject to natural fecundability, having dropped both $X$ and $Y$.

As before,

$$
k_{x}=\left(1-d_{x}{ }^{\prime}\right)^{A}
$$

Evidently,

$$
\begin{aligned}
k_{x y}= & d_{x}{ }^{\prime}\left(1-d_{y}{ }^{\prime}\right)^{A-1}+\left(1-d_{x}{ }^{\prime}\right) d_{x}{ }^{\prime}\left(1-d_{y}{ }^{\prime}\right)^{A-2}+\cdots \\
& +\left(1-d_{x}{ }^{\prime}\right)^{A-2} d_{x}{ }^{\prime}\left(1-d_{y}{ }^{\prime}\right)+\left(1-d_{x}{ }^{\prime}\right)^{A-1} d_{x}{ }^{\prime} \\
= & \sum_{i=0}^{A-1}\left(1-d_{x}{ }^{\prime}\right)^{i} d_{x}{ }^{\prime}\left(1-d_{y}{ }^{\prime}\right)^{A-i-1}
\end{aligned}
$$

To drop both $X$ and $Y$ during the infecundable period presupposes that $X$ is dropped at the end of some month $1,2, \ldots, A-1$. If $X$ is dropped at the end of month $m, 1 \leqslant m \leqslant A-1$, the probability of dropping $Y$ by end of month $A$ is the complement of not dropping it by then, or

$$
1-\left(1-d_{y}{ }^{\prime}\right)^{A-m} \text {. }
$$




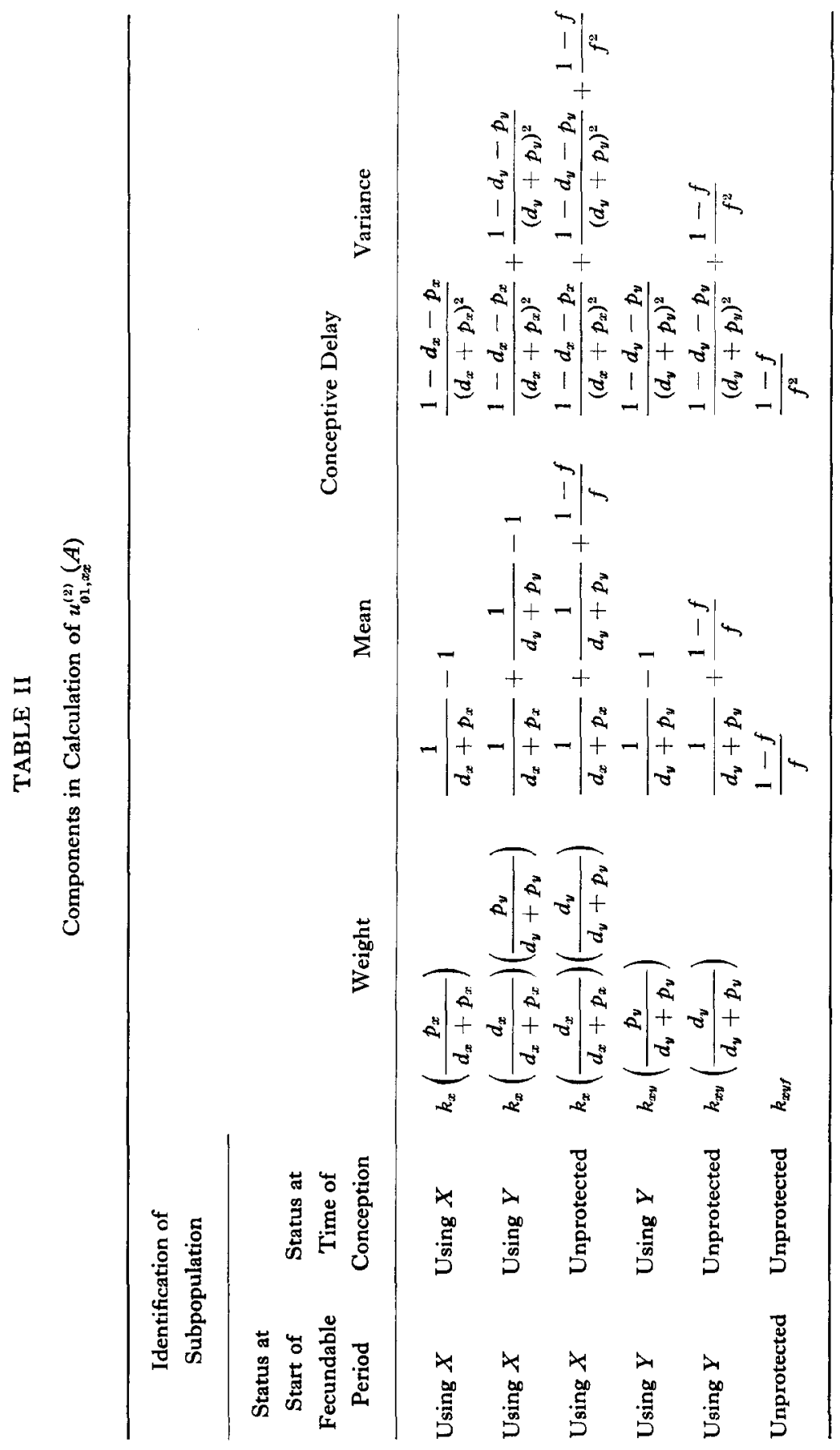


Accordingly,

$$
\begin{aligned}
k_{x y f}= & d_{x}{ }^{\prime}\left(1-\left(1-d_{y}{ }^{\prime}\right)^{A-1}\right)+\left(1-d_{x}{ }^{\prime}\right) d_{x}{ }^{\prime}\left(1-\left(1-d_{y}{ }^{\prime}\right)^{A-2}\right)+\cdots \\
& +\left(1-d_{x}{ }^{\prime}\right)^{A-2} d_{x}{ }^{\prime}\left(1-\left(1-d_{y}{ }^{\prime}\right)\right) \\
= & \sum_{i=0}^{A-2}\left(1-d_{x}{ }^{\prime}\right)^{i} d_{x}{ }^{\prime}\left(1-\left(1-d_{y}{ }^{\prime}\right)^{A-i-1}\right) .
\end{aligned}
$$

Weights, means, and variances are given for each of the six subpopulations in Table II. The mean delay for the total population $u_{01, x y}(A)$ is obtained by summing the products of weights and mean delays of the six subpopulations. The second moment around the origin $\boldsymbol{u}_{01, x y}^{(2)}(A)$ is obtained by summing the products of weights and second moments around the origin of the subpopulations, these second moments each being calculated as the sum of the variance and mean squared. As before we obtain $\sigma_{01, x y}^{2}(A)$ as the difference of $u_{01, x y}^{(2)}(A)$ minus the square of $u_{01, x y}(A)$. When $A=0$, the number of nonzero weights reduces to three and it is easily shown that

$$
u_{01, x y}(0)=\frac{1-p_{x}}{d_{x}+p_{x}}+\frac{d_{x}}{d_{x}+p_{x}} u_{01, y}(0) .
$$

If $A>0$, we have

$$
u_{01, x y}(A)=k_{x} u_{01, x y}(0)+k_{x y} u_{01, y}(0)+k_{x y f} u_{01, f} .
$$

\section{Repetitive Use of Contraception}

In order that the model above still qualify as a renewal process, it is necessary to posit a repetitive family planning strategy, such that, with the exception of the first pregnancy interval in an augmented process, the same effectiveness of contraception applies to all pregnancy intervals. Homogeneity in time and among women must extend to contraceptive practice as well as to fecundity.

When a method $X$ is used repetitively in successive pregnancy intervals we can most meaningfully think of $X$ as always being initiated right after a pregnancy termination or else always at the start of the fecundable period. The latter case eliminates overlap between contraception and anovulation while the former case maximizes overlap. It greatly simplifies formulas (by eliminating an extra summation) if the anovulatory periods following a particular pregnancy outcome are treated as constant. Specifically,

$$
T_{i 0}=u_{i 0} \quad \text { for } \quad i=2,3,4 .
$$


Actually, when the following two conditions are both postulated: (1) Contraception is always initiated either at the start of the first fecundable month or right after a pregnancy, and (2) anovulatory periods following a pregnancy of a given outcome are constant, our notation may be streamlined by putting

$$
u_{01, y}(0)=u_{01,0 y}
$$

and

$$
u_{01, y}\left(u_{i 0}\right)=u_{01, i y}, \quad i=2,3,4
$$

Formulas for the means and variances of birth and pregnancy intervals have been obtained by following the proofs of Perrin and Sheps, at each step making the appropriate modifications to allow for the possibility that contraception may be discontinued for other reasons besides pregnancy. In the case of contraception started at the beginning of the fecundable period, the expected birth and pregnancy intervals are

$$
u_{44, x}=\frac{1}{\theta_{4}}\left\{u_{01,0 x}+\sum_{i-2}^{4} \theta_{i} \eta_{i}\right\}
$$

and

$$
u_{11 . x}=u_{01,0 x}+\sum_{i=2}^{4} \theta_{i} \eta_{i} .
$$

These two formulas are identical with those derived by Perrin and Sheps except that $u_{01,0 x}$ replaces their $u_{01}$. The same parallelism applies to the variances, it being sufficient to substitute $\sigma_{01,0 x}^{2}$ for their $\sigma_{01}^{2}$.

In the case of contraception initiated directly after a pregnancy termination, when following the proof of Perrin and Sheps, care must be taken to allow for the fact that $u_{01,4 x}<u_{01,3 x}<u_{01,2 x}$. Here

$$
u_{44, x}=\frac{1}{\theta_{4}}\left\{\sum_{i=2}^{4} \theta_{i}\left(u_{01, i x}+\eta_{i}\right)\right\}
$$

and

$$
u_{11, x}=\sum_{i=2}^{4} \theta_{i}\left(u_{01, i x}+\eta_{i}\right)
$$

Respccting variances,

$$
\sigma_{11, x}^{2}=\sum_{i=2}^{4} \theta_{i}\left(\sigma_{01, i x}^{2}+\lambda_{i}{ }^{2}\right)+\sum_{i<j} \theta_{i} \theta_{j}\left(u_{01, i x}-u_{01, j x}+\eta_{i}-\eta_{j}\right)^{2}
$$


and

$$
\begin{aligned}
\sigma_{44, x}^{2}= & \lambda_{4}{ }^{2}+\sigma_{01,4 x}^{2}+\frac{\theta_{2}+\theta_{3}}{\theta_{4}} \operatorname{Var}\left(T_{11 . \overline{4} x}\right)+\frac{\theta_{2}+\theta_{3}}{\theta_{4}{ }^{2}}\left[E\left(T_{11 . \overline{4} x}\right)\right]^{2}, \\
E\left(T_{11 . \overline{4} x}\right)= & \frac{\theta_{2}}{\theta_{2}+\theta_{3}}\left(\eta_{2}+u_{01,2 x}\right)+\frac{\theta_{3}}{\theta_{2}+\theta_{3}}\left(\eta_{3}+u_{01,3 x}\right), \\
\operatorname{Var}\left(T_{11 . \overline{4} x}\right)= & \frac{\theta_{2}}{\theta_{2}+\theta_{3}}\left(\sigma_{01,2 x}^{2}+\lambda_{2}{ }^{2}\right)+\frac{\theta_{3}}{\theta_{2}+\theta_{3}}\left(\sigma_{01,3 x}^{2}+\lambda_{3}^{2}\right) \\
& +\frac{\theta_{2} \theta_{3}}{\left(\theta_{2} \mid \theta_{3}\right)^{2}}\left(u_{01,2 x}-u_{01,3 x}+\eta_{2}-\eta_{3}\right)^{2} .
\end{aligned}
$$

\section{Births Averted}

A segment of contraception averts births indirectly by delaying the next conception, thereby reducing the residual reproductive period when births are a possibility. Two measures of impact of a segment of $X$ are its net delaying of the next conception and the fractional number of births it averts. Both measures are available through suitable comparisons of pairs of augmented renewal processes, one representing an experimental cohort and the other a control cohort. The two cohorts differ only in that the experimental cohort exploits $X$ during an initial pregnancy interval (the "preperiod") while the control cohort does not. Otherwise, the two cohorts are the same. They are identical in fecundity including an equally long residual reproductive period (preperiod plus the succeeding pregnancy intervals that compose the "postperiod"). Both cohorts start the preperiod in the same initial state and during the postperiod both cohorts follow the same repetitive family planning strategy. Hence, any difference between the two cohorts with respect to time of next conception or cumulative births during the residual reproductive period of $T$ months is ascribable to the presence and absence of one segment of $X$ during the preperiod.

Consider the example of a program introducing contraceptive $X$ into a population that otherwise would have practiced no contraception. The net delay of next conception is given by

$$
I_{x, f}=u_{01, x}(A)-u_{01, f}
$$

Given that the residual reproductive period starts with a pregnancy termination and has a total length of $T$ months, $T$ sufficiently large, then without contraception during the preperiod, the postperiod averages

$$
T_{c}=T-\alpha-u_{01, f},
$$


with $\alpha$ denoting the mean number of anovulatory months preceding the first fecundable period. Expected births during the residual reproductive period are

$$
B_{c}=E\left[N_{4}\left(T_{c}\right) \mid S_{1}\right]=\frac{T-\alpha-u_{01, f}}{u_{44, f}}+\frac{u_{44, f}^{(2)}+u_{44, f}}{2 u_{44, f}^{2}}-\frac{u_{14, f}}{u_{44, f}} .
$$

By practice of one segment of $X$ during the preperiod, the postperiod is shortened to a mean of

$$
T_{e}=T-\alpha-u_{01, x}(A)
$$

and expected births are

$$
B_{e}=E\left[N_{4}\left(T_{e}\right) \mid S_{1}\right]=\frac{T-\alpha-u_{01, x}(A)}{u_{44, f}}+\frac{u_{44, f}^{(2)}+u_{44, f}}{2 u_{44, f}^{2}}-\frac{u_{14, f}}{u_{44, f}} .
$$

Births averted per segment of $X$, given by the difference in cumulative births during the two postperiods, are

$$
B=B_{c}-B_{e}=I_{x, f} / u_{44, f} .
$$

From this formula it is evident that under the conditions being assumed, the impact of a segment of $X$ is dependent in part on its shortening of the residual reproductive period, but also in part on the common family planning strategy followed by the two cohorts during the postperiod. This strategy determines the added childbearing to be expected of the experimental cohort had the given foreshortening of its residual reproductive period not taken place.

Another possible effect of a family planning program is to persuade noncontraceptors to adopt $X$ one pregnancy interval earlier than they otherwise would have. In this case, the net delay of next conception is the same, namely $I_{x, f}$. But we have to stipulate repetitive use of $X$ in the postperiod and as a result obtain

$$
B=I_{x, y} / u_{44, x}
$$

When couples are switching between two program contraceptives, from $Y$ to $X$ say, it matters whether $X$ is "supplementing" or "replacing" $Y$. By $X$ supplementing $Y$ is meant that any droppers of $X$ switch back to $Y$ within the same pregnancy interval and the net delay of next conception is

$$
u_{01, x y}(A)-u_{01, y}(A) \text {. }
$$

In contrast, when $X$ replaces $Y$, it is practiced during a pregnancy interval to the exclusion of $Y$ and any droppers of $X$ cease practice of contraception, at least until after the next pregnancy. Hence net delay of next conception is

$$
u_{01, x}(A)-u_{01, y}(A) .
$$


Three contexts are usefully distinguished.

1. The family planning program is introducing new contraception into a community (or perhaps fostering contraception where it has not been practiced before.)

2. The program is persuading couples to start their family limitation earlier.

3. The program is encouraging couples to switch from one program contraceptive to another.

Various possibilities ordered according to these three contexts arc cnumcrated along with formulas in Table III.

TABLE III

Classification of Family Planning Contexts and Respective Formulas for Net Delay of Next Conception and Births Averted

Formulas

Family Planning Context

Net Delay of

Births

Next Conception

Averted

Program introducing new contraception

$X$ in place of no contraception

$Y$ in place of no contraception

$X$ in place of $C$

$Y$ in place of $C$

$X$ introduced as a supplement to $C$

$Y$ introduced as a supplement to $C$

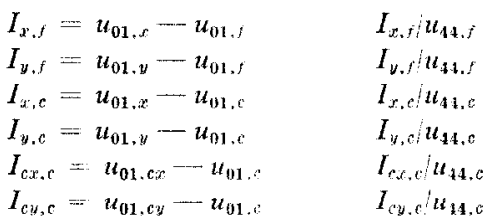

Program hastening family limitation or use of a particular method

Repetitive use of $X$ started one pregnancy interval earlier

Same for $Y$

Shift from non program $C$ to program $X$ one pregnancy interval earlier

Same for $Y$

$$
\begin{aligned}
& I_{x, f}=u_{01, x}-u_{01, f} \quad I_{x, f} / u_{\mathbf{4}, x} \\
& I_{y, f}=u_{01, y}-u_{01, j} \quad I_{y, j} / u_{41, v} \\
& I_{x, c}=u_{01, x}-u_{01, .} \quad I_{x, c} u_{41, x} \\
& I_{y, c}=u_{01, y}-u_{01, c} \quad I_{y, c} / u_{14, y} \\
& I_{x y . y}=u_{01, x y}-u_{01, v} \quad I_{x y, y} / u_{44 . y} \\
& I_{x, y}=u_{01, x}-u_{01, y} \quad I_{x, y} / u_{44, y} \\
& I_{y x, y}=u_{01, y x}-u_{01, y} \quad I_{y, x, y} / u_{44, x} \\
& I_{x, y}=u_{01, x}-u_{01, y} \quad I_{x, y} / u_{44, x}
\end{aligned}
$$

Switches among two program contraceptives

$X$ supplements $Y$ for one interval

$X$ replaces $Y$ for one interval

Repetitive use of $X$ started as a supplement to $Y$

Shift from $Y$ to repetitive use of $X$ started one pregnancy interval earlier 


\section{ApPlications}

The context to which the present model is best fitted in view of its simplifying assumptions is that of young, "normally fecund" acceptors of IUD and oral contraception in the setting of a mass family planning program. In this setting IUD and orals exhibit high effectiveness but appreciable discontinuation rates. Given early acceptance and substantial discontinuation rates, practice of the first segment of program contraception is confined mainly to the first half of the reproductive period when sterility is rare and declines in fecundability slow. As a consequence, the simplifying assumptions of constant fecundability and of everyone conceiving again are made more reasonable.

The following parameters define a rather high fecundity level characteristic of relatively fecund couples early in marriage:

$$
\begin{array}{rll}
f=0.20, & u_{20}=1, & \eta_{2}=3+1=4, \quad \lambda_{i}{ }^{2}=0, \quad i=2,3,4 . \\
\theta_{2}=0.20, & u_{30}=3, & \eta_{3}=9+3=12 \\
\theta_{3}=0.05, & u_{40}=8, & \eta_{4}=9+8=17 \\
\theta_{4}=0.75, & &
\end{array}
$$

As consequences of the above assignments, we have

$$
\begin{array}{rlrl}
u_{01} & =4.0, & \sigma_{01}^{2} & =20.0 \\
\Sigma \theta_{i} \eta_{i} & =14.2, & \\
u_{11}=18.2, & \sigma_{11}^{2}=46.9 \\
u_{44}=24.2, & \sigma_{44}^{2}=71.0 .
\end{array}
$$

When not practicing contraception, these couples can expect to average about one birth every 2 years, a pregnancy every year and a half. The variances of birth and pregnancy intervals are underestimated as a result of setting $\lambda_{i}{ }^{2}=0$, $i=2,3,4$, but provide a standard against which to measure the increased variation introduced by contraceptive practice.

We define three contraceptives. First, taken as representative of IUD, is contraceptive $X$ with characteristics $e_{x}=0.95$ and $d_{x}{ }^{\prime}=0.02846$. In the absence of contraception as a competing risk, a monthly discontinuation rate of 0.02846 leaves $50 \%$ retaining the device at the end of 2 years. In both the Korean and Taiwan programs, 2-yr retention rates have been slightly less than $50 \%$ (Mauldin, Nortman, and Stephan, 1967). The effectiveness of IUD has been estimated as 0.95 in a Taiwan sample (Potter, McCann, and Sakoda, 1970). In mass programs, the antiovular pills have typically exhibited high effectiveness, but also considerably higher discontinuation rates than IUD (Jones and Mauldin, 1967). To represent this case, the second contraceptive $Y$ 
is assigned characteristics $e_{y}=1.00$ and $d_{y}{ }^{\prime}=0.05612$, implying that only $25 \%$ of first segments of pill last as long as 2 -years. The third contraceptive $C$, representing preprogram contraception, is defined by $e_{0}=0.90$ and $d_{c}^{\prime}=0.05612$. The rationale here is that the irregular practice often associated with traditional contraception means relatively low effectiveness and low continuation.

Assigning the parameter $A$ a value of zero means that contraception is initiated at the start of the first fecundable month while $A=8$ is taken to mean that contraception is begun right after a birth. For contraception started right after an abortion or a stillbirth, appropriate values of $A$ are 1 and 3 .

Given these assumptions, the monthly probabilities of accidental pregnancy are $p_{x}=0.01, p_{y}=0.00$, and $p_{c}=0.02$. Even if $p_{x}$ is small, because

$$
d_{x}=\left(1-p_{x}\right) d_{x}{ }^{\prime}=0.99(0.02846)=0.02818
$$

is also small, the proportion eventually conceiving during practice of $X$, conditional to $A=0$, is 0.26 , derived from $p_{x} /\left(d_{x}+p_{x}\right)$; it is 0.21 , if $A=8$, as determined by $k_{x} p_{x} /\left(d_{x}+p_{x}\right)$. In the case of contraceptive $C$, the corresponding proportions take nearly the same values, namely, 0.26 and 0.16 . Naturally if $p_{y}=0.00$, the proportion conceiving during practice of $Y$ is zero.

Table IV provides means and standard deviations of delays to next conception

\section{TABLE IV}

Measures Relating to Use of a Single Segment of Contraception, by Overlap with Anovulation and Characteristics of the Contraceptive

\begin{tabular}{|c|c|c|c|c|c|c|c|}
\hline & & \multicolumn{3}{|c|}{ Overlap $(A=8)$} & \multicolumn{3}{|c|}{ No Overlap $(A=0)$} \\
\hline \multicolumn{2}{|c|}{$\begin{array}{l}\text { Characteristics } \\
\text { of Contraceptive }\end{array}$} & \multirow{2}{*}{$\begin{array}{c}\text { Proportion } \\
\text { Retaining } \\
K_{x}\end{array}$} & \multicolumn{2}{|c|}{$\begin{array}{l}\text { Delay to Next } \\
\text { Conception }\end{array}$} & \multirow{2}{*}{$\begin{array}{l}\text { Proportion } \\
\text { Retaining } \\
\qquad K_{x}\end{array}$} & \multicolumn{2}{|c|}{$\begin{array}{l}\text { Delay to Next } \\
\text { Conception }\end{array}$} \\
\hline$e_{x}$ & $d_{x}^{\prime}$ & & Mean & $\begin{array}{l}\text { Standard } \\
\text { Deviation }\end{array}$ & & Mean & $\begin{array}{l}\text { Standard } \\
\text { Deviation }\end{array}$ \\
\hline 0.95 & 0.02846 & 0.794 & 25.8 & 25.4 & 1.0 & 28.9 & 26.3 \\
\hline 1.00 & 0.05612 & 0.630 & 15.2 & 16.8 & 1.0 & 21.8 & 17.9 \\
\hline 0.90 & 0.05612 & 0.630 & 11.6 & 12.5 & 1.0 & 16.0 & 13.8 \\
\hline
\end{tabular}

when a single segment of contraception is practiced. Note that despite its lower effectiveness, $X$ (IUD) has greater impact than $Y$ (pill) on account of its lower discontinuation rate. The effect of a potential overlap between contraception and anovulation of 8 months is to subtract several months from the mean and roughly one month from the standard deviation of delays to next conception. 
Actually, the effect of overlap is a function of $p_{x}$ and $d_{x}{ }^{\prime}$ as well as $A$. For a given $A$, the effect of overlap will be small if either $d_{x}{ }^{\prime}$ is close to zero or $p_{x}$ is close to $f$. In the former case, few will drop $X$ before entering the fecundable period; in the latter case it scarcely matters whether they are practicing contraception or not.

In Table $\mathrm{V}$ are given the means and standard deviations of delays to next

\section{TABLE V}

Mean and Standard Deviation to Next Conception When Two Contraceptives are Used in the Same Pregnancy Interval, by Overlap with Anovulation and Characteristics of the Two Contraceptives

\begin{tabular}{|c|c|c|c|c|c|}
\hline \multirow{2}{*}{ Two } & \multirow{2}{*}{$\begin{array}{l}\text { Sequence of } \\
\text { Contraceptives } \\
\text { Practiced }^{a}\end{array}$} & \multicolumn{2}{|c|}{$\begin{array}{l}\text { Mean Delay to Next } \\
\text { Conception }\end{array}$} & \multicolumn{2}{|c|}{$\begin{array}{l}\text { Standard Deviation of } \\
\text { Delay to Next Conception }\end{array}$} \\
\hline & & $\begin{array}{l}\text { Overlap } \\
(A=8)\end{array}$ & $\begin{array}{l}\text { No Overlap } \\
\quad(A=0)\end{array}$ & $\begin{array}{r}\text { Overlap } \\
(A=8)\end{array}$ & $\begin{array}{l}\text { No Overlap } \\
\quad(A=0)\end{array}$ \\
\hline & $X, Y$ & 37.2 & 40.0 & 30.7 & 31.6 \\
\hline & $Y, X$ & 39.2 & 46.7 & 31.0 & 31.3 \\
\hline & $C, X$ & 31.3 & 34.3 & 28.1 & 28.9 \\
\hline & $X, C$ & 32.8 & 37.7 & 28.4 & 29.2 \\
\hline & $C, Y$ & 25.1 & $29: 1$ & 21.4 & 22.4 \\
\hline & $Y, C$ & 26.4 & 33.8 & 21.5 & 22.0 \\
\hline
\end{tabular}

${ }^{a} e_{x}=0.95$ and $d_{x}{ }^{\prime}=0.02846 ; e_{c}=0.90 ; d_{c}^{\prime}=0.05612 ; e_{y}=1.00 ; d_{y}^{\prime}=0.05612$.

conception when all possible pairings of $X, Y$, and $C$ are practiced in the same pregnancy interval. There is a slight advantage to practicing first the more effective of the two methods (not necessarily the method with more impact). However, this generalization would not hold if there was typically a long "gap" of nonpractice of contraception between discontinuing the first method and switching to the second. Then one would want the method with greater impact to precede because few discontinuers would survive the gap to use the second method.

As a theoretical extreme, suppose that women are willing to reinsert immediately after losing a device as many times as necessary in a single pregnancy interval. Discontinuation loses its significance and the only cause of termination becomes accidental pregnancy. For contraceptive $X$ we then have $k_{x}=1.0$ and $u_{01, x}(A)=1 / p_{x}=100$. The expected number of reinsertions is the number of trials before a "success" (i.e., accidental pregnancy). Probability of success is $p=p_{x} /\left(d_{x}+p_{x}\right)$ and of failure (i.e., expulsion or removal, calling for another reinsertion) is $q=d_{x} /\left(d_{x}+p_{x}\right)$. In accord with the geometric distribution, the 
expected number of reinsertions is $q \mid p=0.02846 / 0.01=2.85$. Correspondingly, in the case of contraceptive $C, u_{01, c}(A)=50$ and the expected number of segments of $C$ per pregnancy interval would be $0.05612 / 0.02=2.81$.

Net delays of next conception and births averted by one segment of contraception, classified by type of substitution and family planning context, are given in Table VI, based on the formulas of Table III. Several points are illustrated. First, with parameter $A$ fixed, a given contraceptive has maximum

\section{TABLE VI}

Net Delay of Next Conception and Births Averted, by Family Planning Context, by Overlap with Anovulation

\begin{tabular}{|c|c|c|c|c|}
\hline \multirow{2}{*}{$\begin{array}{c}\text { Family } \\
\text { Planning } \\
\text { Context }\end{array}$} & \multicolumn{2}{|c|}{$\begin{array}{l}\text { Net Delay of } \\
\text { Next Conception }\end{array}$} & \multicolumn{2}{|c|}{ Births Averted ${ }^{a}$} \\
\hline & $\begin{array}{r}\text { Overlap } \\
(A=8)\end{array}$ & $\begin{array}{l}\text { No Overlap } \\
\quad(A=8)\end{array}$ & $\begin{array}{r}\text { Overlap } \\
(A=8)\end{array}$ & $\begin{array}{l}\text { No Overlap } \\
\qquad(A=0)\end{array}$ \\
\hline \multicolumn{5}{|l|}{$\begin{array}{l}\text { Program introducing new } \\
\text { contraception }\end{array}$} \\
\hline$X$ in place of no contraception & 19.8 & 24.9 & 0.82 & 1.03 \\
\hline$Y$ in place of no contraception & 11.2 & 17.8 & 0.46 & 0.74 \\
\hline$X$ in place of $C$ & 12.2 & 12.9 & 0.34 & 0.32 \\
\hline$Y$ in place of $C$ & 3.7 & 5.8 & 0.10 & 0.15 \\
\hline$X$ introduced as a supplement to $C$ & 19.8 & 18.2 & 0.56 & 0.45 \\
\hline$Y$ introduced as a supplement to $C$ & 13.6 & 13.1 & 0.38 & 0.33 \\
\hline \multicolumn{5}{|l|}{$\begin{array}{l}\text { Program hastening family limitation or } \\
\text { use of a particular method }\end{array}$} \\
\hline $\begin{array}{l}\text { Repetitive use of } X \text { started one } \\
\text { pregnancy interval earlier, }\end{array}$ & 19.8 & 24.9 & 0.38 & 0.43 \\
\hline Same for $Y$ & 11.2 & 17.8 & 0.27 & 0.37 \\
\hline $\begin{array}{l}\text { Shift from } C \text { to } X \text { one pregnancy } \\
\text { interval earlier, }\end{array}$ & 12.2 & 12.9 & 0.24 & 0.22 \\
\hline Same for $Y$ & 3.7 & 5.8 & 0.09 & 0.12 \\
\hline \multicolumn{5}{|l|}{$\begin{array}{l}\text { Switches among two program contra- } \\
\text { ceptives }\end{array}$} \\
\hline$X$ supplements $Y$ for one interval & 22.0 & 20.2 & 0.54 & 0.42 \\
\hline$X$ replaces $Y$ for one interval & 8.5 & 7.1 & 0.21 & 0.15 \\
\hline $\begin{array}{l}\text { Repetitive use of } X \text { started as a } \\
\text { supplement to } Y\end{array}$ & 23.9 & 24.9 & 0.46 & 0.43 \\
\hline $\begin{array}{l}\text { Shift from } Y \text { to repetitive use of } X \\
\text { started one pregnancy interval } \\
\text { earlier }\end{array}$ & 8.5 & 7.1 & 0.16 & 0.12 \\
\hline
\end{tabular}

$a_{u_{44, S}}=24.2$ and according as $A=0$ or $8, u_{14, \infty}=57.4,51.9 ; u_{14, y}=48.0,40.9$; and $u_{44, c}=40.2,35.5$. 
impact in the case of nonsubstitution. Its impact is less when it is substituting for another contraceptive or when it is merely being adopted one pregnancy interval earlier. Second, a contraceptive $X$ has more impact when supplementing $Y$ than when replacing it. This difference is accentuated when the ratio $d_{x} /\left(d_{x}+p_{x}\right)$ is close to 1.0 and the impact of $Y$ is large. Third, when $X$ is substituting for another method $Y$, the effect of a longer potential overlap between contraception and anovulation (e.g., $A=8$ instead of 0 ) may be to increase both the net delay of next conception $I_{x, y}$ and births averted. This paradoxical result obtains when the expected delay to next conception under $Y$ is more penalized by the longer potential overlap than the corresponding expected delay under $X$. Also, the expected birth interval $u_{44, y}$ is reduced by longer potential overlap.

Fourth, and perhaps the most interesting result, on account of the appreciable discontinuation rates stipulated, births averted by one segment of contraception is generally well under 1.0. Indeed, even when one combines $X$ (IUD) and its relatively low discontinuation rate with an otherwise optimal set of conditionscontraception introduced into a community where it has not existed before, no overlap with anovulation, high fecundity, no sterility, and no decline in fecundity as a function of ageing--one still estimates only one birth averted by a segment of contraception.

A question closely related to the last point is: For a given contraceptive effectiveness $e$, what discontinuation rate $d_{0}{ }^{\prime}$ is required in order to avert exactly one birth. In the case of $X$ being introduced into a community innocent of

\section{TABLE VII}

Discontinuation Rate Necessary to Avert One Birth, According to Effectiveness of Program Contraceptive and Characteristics of the Contraceptive It Is Replacing ${ }^{a}$

\begin{tabular}{|c|c|c|c|c|}
\hline \multirow{2}{*}{$\begin{array}{l}\text { Effectiveness of } \\
\text { Program } \\
\text { Contraceptive }\end{array}$} & \multirow{2}{*}{$\begin{array}{c}\text { Characteristics of } \\
\text { Contraceptive } \\
\text { Being } \\
\text { Replaced }\end{array}$} & \multirow{2}{*}{$\begin{array}{c}\text { Required } \\
\text { Drop } \\
\text { Out } \\
\text { Rate } \\
d_{0}^{\prime}\end{array}$} & \multicolumn{2}{|c|}{$\begin{array}{l}\text { Proportion of Women Continuing } \\
\text { Contraception Two Years, } \\
\text { Given } d_{0}^{\prime}\end{array}$} \\
\hline & & & $\begin{array}{c}\text { In Absence of } \\
\text { Pregnancy }\end{array}$ & $\begin{array}{l}\text { In Presence of } \\
\text { Pregnancy }\end{array}$ \\
\hline "IUD" (e $\left.e_{x}=0.95\right)$ & $\begin{array}{l}e_{y}=0.0 \\
e_{c}=0.90 ; d_{c}^{\prime}=0.05612 \\
e_{y}=1.00 ; d_{y}^{\prime}=0.05612\end{array}$ & $\begin{array}{l}0.02308 \\
0.01420 \\
0.01142\end{array}$ & $\begin{array}{l}0.57 \\
0.71 \\
0.76\end{array}$ & $\begin{array}{l}0.49 \\
0.60 \\
0.65\end{array}$ \\
\hline "Pill" $\left(e_{y}=1.00\right)$ & $\begin{array}{l}e_{f}=0.0 \\
e_{t}=0.90 ; d_{t}^{\prime}=0.05612 \\
e_{x}=0.95 ; d_{x}^{\prime}=0.02846\end{array}$ & $\begin{array}{l}0.03188 \\
0.02334 \\
0.01681\end{array}$ & $\begin{array}{l}0.46 \\
0.57 \\
0.67\end{array}$ & $\begin{array}{l}0.46 \\
0.57 \\
0.67\end{array}$ \\
\hline
\end{tabular}

${ }^{a}$ Assuming that contraception is initiated 8 months before end of anovulation. 
contraception, it is required that $I_{x, f}=u_{44, f}$. If $X$ is replacing repetitive use of another method $Y$, then $I_{x, y}$ must equal $u_{44, y}$. Some illustrative values are given in Table VII, derived by an iterative procedure. ${ }^{2}$ In the last two columns of Table VII, $d_{0}{ }^{\prime}$ values are converted into proportions continuing $X$ for 2 years. The formulas used for this purpose are $\left(1-d_{0}{ }^{\prime}\right)^{24}$ and $\left(1-d_{0}{ }^{\prime}\right)^{A}\left(1-p_{x}-d_{0}{ }^{\prime}\right)^{24-A}$.

As a last application, it is worth demonstrating that the repetitive use of $X$ alone, much less $Y$ or $C$ alone, during a residual reproductive period of 10 or 15 years, results in a considerable expectation of excess births. To be conservative, assume that the residual reproductive period starts with a birth and that contraceptive $X$ is always initiated at the most favorable time, namely, start of the first fecundable month. Then from

$$
E\left[N_{4}(T) \mid S_{4}\right]=\frac{T}{u_{44, x}}+\frac{u_{44 . x}^{(2)}+u_{44, x}}{2 u_{44, x}^{2}}-1,
$$

we have 1.82 births expected in a 10 -year period $(T=120)$ and 2.97 in a 15 -year residual reproductive period $(T=180)$. Of course the failure to allow for declining fecundity with advancing age leads to a somewhat exaggerated estimate of excess fertility. The present model is less realistic about the second half of the reproductive period than the first. ${ }^{3}$

\section{ACKNOWLEDGMENTS}

The writer gratefully acknowledges support received from the Ford Foundation and The Population Council, the programming assistance of Barbara McCann, and the helpful comments of Anrudh K. Jain.

\section{REFERENCES}

Jones, G. W. AND Mauldin, W. P. 1967. Use of oral contraceptives with special reference to developing countries, Studies in Family Planning, No. 24, 1-12.

Mauldin, W. P., Nortman, D., and Stephan, F. F. 1967. Retention of IUDs: An international comparison, Studies in Family Planning No. 18, 1-12.

Perrin, E. B. AND ShePs, M. C. 1964. Human reproduction: A stochastic process, Biometrics 20, 28-45.

${ }^{2}$ Interestingly enough, in the case of nonsubstitution, $d_{0}{ }^{\prime}$ is independent of $A$.

${ }^{3}$ For a discussion of this point, see Jain, A. K. 1968. Predicting duration-specific averages and variances of live births: Application of a stochastic model of human reproduction, 1968 Social Statistics Section Proc. Amer. Statist. Ass. 248-255. 
Potter, R. G. 1969. Renewal theory and births averted, International Union for the Scientific Study of Population: General Conference, London, September, 1969, 6 pp. Potter, R. G., MCCANN, B., and SaKoda, J. M. 1970. Selective fecundity and measurement of contraceptive effectiveness, Milbank Memorial Fund Quart. 48, 96-99.

ShEPS, M. C. 1963. Changes in birth rates as a function of contraceptive effectiveness: Some applications of a stochastic model, Amer. J. Public Health 53, 1031-1046.

Sheps, M. C., MenKen, J. A., ANd Radick, A. P. 1969. Probability models for family building: An analytical review, Demography 6, 161-184. 